\title{
Non-empirical double-hybrid functionals: an effective tool for chemists
}

Eric Brémond ${ }^{1}$, Ilaria Ciofini ${ }^{2}$, Juan Carlos Sancho-García ${ }^{3}$ and Carlo Adamo ${ }^{1,2,4, *}$

${ }^{1}$ CompuNet, Istituto Italiano di Tecnologia, via Morego 30, I-16163 Genoa, Italy.

${ }^{2}$ Institut de Recherche de Chimie Paris, PSL Research University, CNRS, Chimie ParisTech, 11 rue Pierre et Marie Curie, F-75005 Paris, France.

${ }^{3}$ Departamento de Química Física, Universidad de Alicante, E-03080 Alicante, Spain.

${ }^{4}$ Institut Universitaire de France, 103 Boulevard Saint Michel, F-75005 Paris, France.

\section{Conspectus}

Density Functional Theory (DFT) emerged in the last two decades as the most reliable tool the description and prediction of properties of molecular systems and extended materials, coupling in an unprecedented way high accuracy and reasonable computational cost. This success rests also on the development of more and more performing Density Functional Approximations (DFAs). Indeed, the Achilles' heel of DFT is represented by the exchange-correlation contribution to the total energy, which, being unknown, must be approximated. Since the beginning of the '90s, global hybrids (GH) functionals, where an explicit dependence of the exchange-correlation energy on

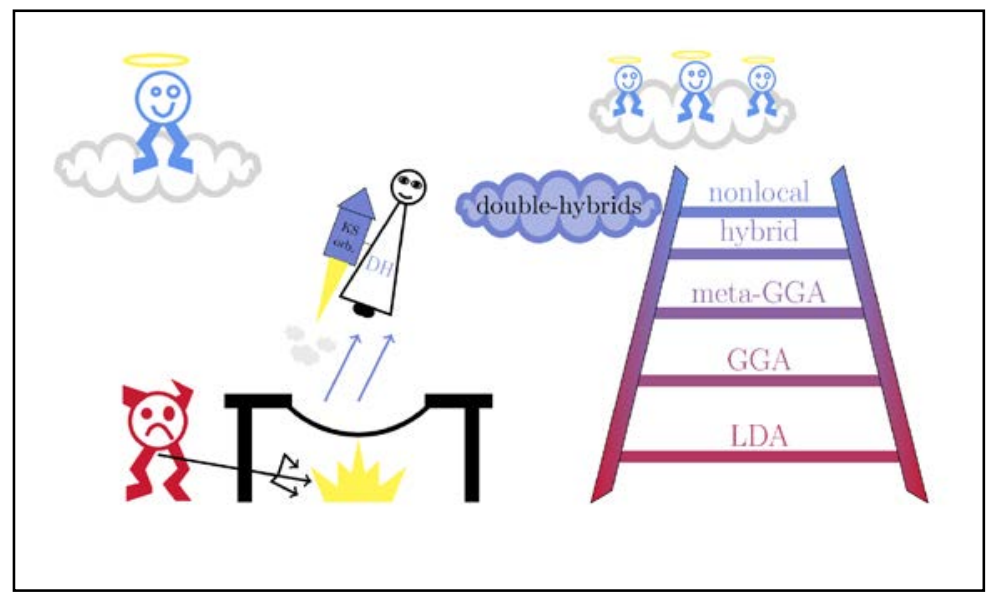
occupied Kohn-Sham orbitals is introduced thanks to a fraction of Hartree-Fock like exchange, imposed themselves as the most reliable DFAs for chemical applications. However, if these functionals normally provide results of sufficient accuracy for most of the cases analyzed, some properties, such as thermochemistry or dispersive

interactions, can still be significantly improved. A possible way out is represented by the inclusion, into the exchange-correlation functional, of an explicit dependence on virtual Kohn-Sham orbitals via Perturbation Theory. This leads to a new class of functionals, called double-hybrids (DHs). In this Account, we describe our non-empirical approach to DHs, which, following the line traced by the Perdew-Burke-Erzerhoh approach, allows for the definition of a GH (PBE0) and a DH (QI-DH) model. In such a way, a whole family of non-empirical functionals, spanning on the highest rungs of the Perdew's quality scale, is now available and competitive with other -more empirical- DFAs. 
Discussion of selected cases, ranging from thermochemistry and reactions to weak interactions and excitations energies, not only show the large range of applicability of non-empirical DFAs, but also underline how increasing the number of theoretical constrains parallel with an improvement of the DFA's numerical performances. This fact further consolidates the strong theoretical framework of non-empirical DFAs.

Finally, even if non-empirical DH approaches are still computationally expensive, relying on the fact that they can benefit of all technical enhancements developed for speeding-up post-HartreeFock methods, there is a substantial hope for their near future routine application to the description and prediction of complex chemical systems and reactions.

1) IIT; 2) Chimie ParisTech ; 3) Universidad de Alicante ; 4) IUF *corresponding author : carlo.adamo@chimie-paristech.fr 


\section{Introduction}

Among electronic structure methods, Density Functional Theory (DFT) represents an invaluable tool for the description and prediction of properties of molecular and extended systems. Its success is mainly related to the development, in the framework of the Kohn-Sham (KS) approach, of several Density Functional Approximations (DFAs) of increasing accuracy and moderate computational cost. Indeed, if many new exchange-correlation density-functionals belonging to the highest rungs of the Perdew ladder ${ }^{1}$ have been developed, the quest of more accurate functionals for the description of a wider variety of properties still remains a major research challenge.

Overall, global- and/or range-separated hybrid functionals, which are characterized by an explicit dependence of the exchange-correlation energy on occupied KS orbitals thanks to the inclusion of a fraction of Hartree-Fock (HF) like exchange (such as PBE0, ${ }^{2,3}$ B3LYP, ${ }^{4-6}$ CAM-B3LYP, ${ }^{7}$ or LC$\omega \mathrm{PBE}^{8}$ ), present a very good performance to computational time ratio. For this reason, these functionals were and are still intensively applied to model a number of ground- and excited-state properties and phenomena.

If the use of hybrid functionals normally provides results of sufficient accuracy for most of the cases analyzed, other properties, including thermochemistry and weak interactions, could be still significantly improved. In order to correctly describe a larger number of properties and, more generally, to better describe long-range electron correlation, density-functionals including an explicit dependence on virtual KS orbitals were more recently developed. ${ }^{9-11}$

Actually, early attempts devoted to include self-consistently a dependence on virtual KS orbitals via Perturbation Theory (PT), firstly introduced by Levy and Görling, ${ }^{12}$ were computationally demanding while providing no substantial improvement with respect to standard approaches, at least in the case of benchmark cases. ${ }^{13}$ Nonetheless, pioneering works ${ }^{14,15}$ allowed to foresee more efficient ways of including the PT contribution, leading to a new class of functionals belonging to the fifth rung of the Perdew ladder: ${ }^{1}$ the so called double-hybrid (DH) density-functionals.

From 2006, DHs were further and extensively developed by Grimme. ${ }^{16}$ Borrowing the statement of Bartlett and collaborators, ${ }^{17}$ these DFAs are somehow based on the "best of both worlds" strategy. The total exchange and correlation energy in a $\mathrm{DH}$ can be defined as:

$$
E_{x c}^{D H}[\rho]=a_{x} E_{x}^{H F}+\left(1-a_{x}\right) E_{x}^{D F A}[\rho]+\left(1-a_{c}\right) E_{c}^{D F A}[\rho]+a_{c} E_{c}^{M P 2} .
$$

where $E_{x}^{H F}$ represents the HF-like exchange, $E_{x}^{D F A}$ and $E_{c}^{D F A}$ stand for the semilocal exchange and correlation energies and $E_{c}^{M P 2}$ is a second-order Møller-Plesset (MP2) correlation energy term. The first three terms are computed in a self-consistent fashion following a standard KS approach, while 
the latter term $\left(E_{c}^{M P 2}\right)$ is added a posteriori, and generally evaluated from the KS orbitals obtained self-consistently. Nonetheless, orbital-optimized (OO) approaches have been recently proposed in literature. ${ }^{18-19}$

Several DHs were developed with the aim of enhancing both DFT and Wave Function Theory (WFT) based methods' accuracy by their synergic combination. They differ either for the type of the semilocal functional used, or in the way the MP2 contribution is considered. For instance, the inclusion of a same spin or spin-opposite scaling of the MP2 contribution was recently proven to ameliorate the performance. ${ }^{20-23}$ Analogously the use of a perturbative term computed from B3LYP, PBE0 or purposely optimized KS orbitals yield excellent results. ${ }^{23,24}$ All these DHs share a common feature: the presence of at least two, empirically defined, parameters, i.e. $a_{x}$ and $a_{c}$, fitted on specific training sets.

More recently, several authors tried to rationally and non-empirically define the relationship existing between these two parameters. Toulouse et al., making use of the Adiabatic Connection (AC) formalism ${ }^{25}$, derived a series of one-parameter DHs, and pointed out the existence of a quadratic $\left(a_{c}=a^{2}{ }_{x}\right)$ or cubic $\left(a_{c}=a_{x}^{3}\right)$ relationship between $a_{x}$ and $a_{c}{ }^{26,27}$ Fromager proposed a two parameters DH formalism relying on a $a_{c} \leq a^{2}{ }_{x}$ relation. ${ }^{28}$ Nonetheless, the value of $a_{x}$ and $a_{c}$ is needed to define new, non-empirical DH. Working along this line, we have developed a number of non-empirical DHs based on the AC theorem, where the $a_{x}$ and $a_{c}$ value directly stems from purely theoretical considerations. ${ }^{27,29,30}$ Such functionals are competitive with their parametrized counterparts, ${ }^{31}$ and allow to define a whole family of non-empirical functionals which spans over the highest three rungs of the Perdew ladder. ${ }^{1}$

The aim of this paper is to illustrate such a contribution to the field of non-empirical DHs through a critical discussion of their performance in the modeling of chemically relevant systems. In particular, the behavior of non-empirical $\mathrm{DH}$ density-functionals will be compared to that of a parameterized DH (B2-PLYP), ${ }^{16}$ and to that of standard functionals, nowadays in common use for chemical applications. Our aim is to show the real (qualitative and quantitative) advantages in using DHs and, more particularly non-empirical DH.

\section{Theory}

The generalized gradient approximation (GGA) density-functional developed by Perdew, Burke and Ernzerhof $(\mathrm{PBE})^{32}$ is undoubtedly the best representative non-empirical functional, being based only on selected theoretical constraints that an ideal DFA should respect. This approach paved the way to the development of more complex approximations such as TPSS, ${ }^{33}$ a non-empirical meta- 
GGA, or the global-hybrid (GH) $\mathrm{PBE0}^{2,3}$ rooted in the AC model. ${ }^{25}$ Starting by the definition of the exchange-correlation contribution, $E_{x c}$, to the total KS energy defined as:

$E_{x c}[\rho]=\int_{0}^{1} E_{x c, \lambda}[\rho] d \lambda$,

where the coupling integrand $E_{x c, \lambda}$, is defined such as:

$E_{x c, \lambda}=\left\langle\Psi_{\lambda}\left|V_{e e}\right| \Psi_{\lambda}\right\rangle-\frac{1}{2} \iint d r^{\prime} d r \frac{\rho(r) \rho\left(r^{\prime}\right)}{\left|r-r^{\prime}\right|}$

with $\Psi_{\lambda}$, the wave-function minimizing:

$\left\langle\Psi_{\lambda}\left|T+\lambda V_{e e}\right| \Psi_{\lambda}\right\rangle$,

under the constraint of producing a given density $\rho$ and $V_{e e}$ is the electron-electron interaction potential. In equation (2), $\lambda=0$ corresponds to the KS noninteracting system where the HF exchange (computed using the KS orbitals) is dominant:

$E_{x c, \lambda=0}=E_{x}^{H F}$,

while the real interacting system is obtained for $\lambda=1$. This latter is at the best described by a given DFA:

$E_{x c, \lambda=1}=E_{x c}^{D F A}$.

Different approaches were proposed to solve the integral in eq. (2), as, for instance, that leading to the Becke Half\&Half functional. ${ }^{34}$ Perdew and co-workers proposed a polynomial of the coupling parameter $\lambda$ as integrand of equation (2), which leads to the following expression: ${ }^{35}$

$E_{x c, \lambda}^{h y b}=E_{x c, \lambda}^{D F A}+\left(E_{x}^{H F}+E_{x}^{D F A}\right)(1-\lambda)^{n-1}$,

where the integer $n$ determines how fast the correction to DFA (second term of equation 7) vanishes for $\lambda \rightarrow 1$. Upon integration the exchange and correlation energy is thus defined as :

$E_{x c}^{h y b}=\int_{0}^{1} E_{x c, \lambda}^{h y b} d \lambda=E_{x c}^{D F A}+\frac{1}{n}\left(E_{x}^{H F}-E_{x}^{D F A}\right)$.

Starting from this expression the global-hybrid functional PBE0 can be obtained setting $n=4$ on the basis of perturbation theory: $:^{2,3,35,36}$

$E_{x c}^{P B E 0}=E_{x c}^{P B E}+\frac{1}{4}\left(E_{x}^{H F}-E_{x}^{P B E}\right)$.

PBE0 is thus the first non-empirical GH. Despite of the fully theoretical approach used to hybridize it, this functional is comparable in accuracy to B3LYP, when not better for specific properties. ${ }^{37-40}$. Figure 1 shows the impact of the exact-exchange fraction on the accuracy for the atomization energy of the G2-148 dataset. ${ }^{41}$ Indeed, the $1 / 4$ factor of PBE0, is very close to the minimum. Very 
recently, another dependence of the integrand on $\lambda$ was proposed, leading to a different integer $n$ (such as $n=3)^{42}$

The AC model provides also a formal framework for the development of DHs. Indeed, at the weak interaction limit $(\lambda \rightarrow 0)$, the first-order derivative of the integrand of equation (2) is the secondorder Görling-Levy (GL2) correlation energy, that is :

$$
\left.\frac{\partial E_{x c, \lambda}}{\partial \lambda}\right|_{\lambda=0}=2 E_{c}^{G L 2},
$$

where

$$
E_{c}^{G L 2}=E_{c}^{M P 2}+E_{c}^{\Delta H F} .
$$

The $E_{c}^{\triangle H F}$ contribution is usually though not always ${ }^{43}$ negligible, so that:

$E_{c}^{G L 2} \approx E_{c}^{M P 2}$,

In other words, this class of DHs satisfies all theoretical constraints defined for their parent GGA (PBE) and global-hybrid (PBE0) functionals, plus the Görling-Levy limit. As it will be pragmatically illustrated in the following, the increase of theoretical constraints induces a significant improvement of the performance, and allows the definition of a complete family of nonempirical DFAs. $^{44}$

In order to have an operational formula, the $\lambda$-dependency in equation (2) must be worked out. A simple way is to use an analytical expression of $\lambda$, the simplest consisting in a quadratic polynomial: ${ }^{45}$

$$
E_{x c, \lambda}=a[\rho]+b[\rho] \lambda+c[\rho] \lambda^{2},
$$

where $a$ and $b$ are determined by equations 5 and 6 , while $c$ is determined by the behavior of the integrand (2) close to the upper integral limit. Named Quadratic Integrand double-hybrid (QIDH), ${ }^{30}$ the general form for this model is:

$$
E_{x c}^{Q I D H, \lambda_{x}}=\frac{\lambda_{x}+2}{3} E_{x}^{H F}+\frac{1-\lambda_{x}}{3} E_{x}^{D F A}+\frac{1}{3} E_{c}^{M P 2}+\frac{2}{3} E_{c}^{D F A} .
$$

The fraction of exact-exchange, modulated by the $\lambda_{x}$ parameter, is determined according to the linear-scaled one-parameter DH expression: ${ }^{27}$

$$
E_{x c}^{L S 1 D H, \alpha}=\alpha E_{x}^{H F}+(1-\alpha) E_{x}^{D F A}+\alpha^{3} E_{c}^{M P 2}+\left(1-\alpha^{3}\right) E_{c}^{D F A},
$$

where the fraction of MP2 correlation $\left(a_{c}=\alpha^{3}\right)$ has a cubic dependence on the fraction of exactexchange $\left(a_{x}=\alpha\right)$ so that in equation (14) :

$\lambda_{x}=3^{2 / 3}-2$. 
Another way to obtain a parameter-free $\mathrm{DH}$ is to evaluate equation (15) with a two-point approximation $(\alpha=1 / 2)$, and to set PBE as density-functional approximation. This leads to the PBE0-DH functional: ${ }^{29}$

$$
E_{x c}^{P B E 0-D H}=\frac{1}{2} E_{x}^{H F}+\frac{1}{2} E_{x}^{P B E}+\frac{1}{8} E_{c}^{M P 2}+\frac{7}{8} E_{c}^{P B E} .
$$

From Figure 1, which illustrates the influence of the relative amount of the exact-exchange and of the PT2 correlation contribution on the performances of the given DH on the G2 atomization dataset, ${ }^{41}$ it can be noticed how non-empirical functionals perform very well, all lying close to the belt defined by lowest deviations. Subsequent works introduced system dependency (PBE$\mathrm{ACDH})^{46}$ or fitting procedure (PBE-QACF-2) ${ }^{47}$ and provide similar errors on standard databases of reactions.

\section{Databases and Case Studies}

One of the most important steps in the development of new density-functionals is the validation, also called benchmarking. This procedure consists in measuring the accuracy of the newly developed DFAs in the prediction of selected properties gathered in dedicated datasets. Reference values are sometimes derived from experiment or, more often, computed using correlated WFT methods.

In case of parameterized density-functionals, the databases are generally split into a training and a test set. Since the two sets have weak chemical diversity, the performances obtained on these datasets are often predictable.

More challenging is the case of non-empirical DF, since the validation step reveals if the chosen physical model allows describing (qualitatively and quantitatively) different properties and chemical systems. This step needs the set up of extensive databases.

In this domain, the $\mathrm{Gn}$ datasets for atomization and general thermochemistry are the first examples ${ }^{41,48}$ while, more recently, Truhlar and co-workers further contributed with data concerning kinetics. ${ }^{49}$ Hobza and collaborators focused, instead, on non-covalent interactions. ${ }^{50,51}$ This list is far to be exhaustive but reflects the work of the main actors in the field.

In this work we will make use of the GMTKN30 database ${ }^{52}$ which can be considered as the current state-of-the-art dataset, since it contains a large pool of reactions probing several properties, such as atomization and decomposition energies, self-interaction errors, kinetics, chemical reactions, noncovalent interactions and conformer stabilities. 
Besides providing the errors computed on each of these datasets, giving a flavor of the average performances of a DFA, we will also analyze for the different class of reactions or interactions a case study of chemical relevance, directly extracted from GMTKN30.

Finally, this set has been integrated with some examples concerning excited states, taken from a recently developed dataset for vertical excitations. ${ }^{53}$

\subsection{Main-Group Thermochemistry}

Figure 2 compares the performances of four DHs on main-group thermochemistry datasets with those of their respective GGA and GH parent functionals. All the examined DHs (PBE0-DH, ${ }^{29}$ PBE-QIDH, ${ }^{30}$ TPSS-QIDH $^{30}$ and B2-PLYP ${ }^{16}$ ) possess between 50 and 70\% of HF-like exchange, and between 10 and 30\% of nonlocal PT2 correlation. Atomization and decomposition processes considered deal with covalent bond dissociation, and the electron-electron coulomb repulsion is strong and claims for a correct treatment of dynamic correlation.

Except for PBE0-DH, adding the PT2 nonlocal correlation leads to a systematic improvement of accuracy with respect to the parent global-hybrid. This improvement is particularly relevant in going from B3LYP to B2-PLYP, but less marked when comparing PBE0 (TPSS0) ${ }^{33,35}$ to PBEQIDH (TPSS-QIDH). An explanation for this observation can found in their different protocol of construction. If all DHs deriving from $\mathrm{BLYP}^{54,55}$ are systematically trained to minimize errors on atomization datasets, the PBE derived functionals are not, so that the lower performances are not unexpected.

Within main-group thermochemistry properties, adiabatic processes, illustrated here by ionization and electron affinity (Figure 2, right side), show indeed how non-empirical double-hybrids can actually behave as well as empirical ones.

\subsection{Self-Interaction Error}

The Self-Interaction Error (SIE) is a recurrent problem in DFT. ${ }^{56}$ This error originates from the only partial cancellation of the spurious electron(s) self-repulsion by the exchange energy. This error is generally, but only partially, overcome by introducing a fraction of exact-exchange in the functional. The simplest system to illustrate the effect of this error is the dissociation profile of the dihydrogen cation (Figure 3). Here, the electronic correlation vanishes due to the presence of a single electron, the HF values becoming thus the reference. Double-hybrid density-functionals which are incorporating between 50 and $70 \%$ of exact-exchange overshoot global-hybrids and semilocal DFA, but still do not entirely cancel the spurious SIE. 
SIE also largely impact multielectronic compounds. The SIE11 subset collects eleven cases particularly prone to self-interaction error. On this dataset, non-empirical DHs outperform standard global-hybrids and GGAs, and practically well compare with the empirical B2-PLYP approach (see Figure 3).

\subsection{Kinetics}

Accuracy in predicting barrier heights is of high interest for chemists working on mechanistic investigations and/or kinetic studies. As an example, in Figure 4, the reaction barriers associated to two $\mathrm{S}_{\mathrm{N}} 2$ reactions are discussed. The best estimates are provided by global- and double-hybrids. Semilocal density-functionals give, on the other hand, much larger errors thus underlining the importance of the inclusion of the exact-exchange.

More generally, on the whole barrier heights database, we observe a marked improvement when going from global- to double-hybrids. This improvement is particularly large in case of nonempirical DHs which outperform B2-PLYP.

\subsection{Reactions Energies}

The performance of different functionals on the prediction of Diels-Alder, bond fragmentation, radical stabilization, isomerization or dimerization reactions (here labelled as 'general chemical reactions') are collectively assessed using a single database as reported in Figure 5. The PBE0-DH, PBE-QIDH and TPSS-QIDH DHs exhibit a mean absolute deviation less than $2 \mathrm{kcal} \mathrm{mol}^{-1}$ on the 235 reactions considered, while their parent global-hybrids only provide a mean error of about 4 $\mathrm{kcal} \mathrm{mol}^{-1}$. This impressive improvement in performance can be easily illustrated by the alkane isomerization reaction of 2,2-dimethylpropane in pentane. Only DHs reach the reference energy with an error between 15 to 35\% depending on the DH considered. Other approaches, such as BLYP, are completely unable to describe this simple reaction with errors reaching up to $90 \%$.

\subsection{Noncovalent Interactions}

The description of noncovalent interactions represents a problem for standard semilocal or hybrid functionals, except for those purposely tailored. ${ }^{57}$ Semilocal approximations are built to locally model electronic processes and their correlation part, particularly important for noncovalent interactions, decreases too rapidly with the inter-electronic distance. DHs could in principle succeed in modeling such systems due to the presence of a nonlocal correlation term.

Figure 6 summarizes the performances of the selected density-functionals in simulating the weak interactions of the GMTKN30 database. Overall functionals belonging to the non-empirical PBE 
family better behave than those of the BLYP family. This statement remains true for DHs which bring a further systematic improvement.

For the subset dealing with hydrogen-bond interactions, semilocal functionals or global-hybrid are able to reproduce the binding character of the interaction as exemplified by the dissociation of the water dimer (Figure 7). All functionals tested succeeded in recovering the binding behavior with an error varying between 2 and 16\%, and all of them tend to the correct dissociation limit. As expected, BLYP-based density-functionals tend to underestimate the interaction. Going from the semilocal BLYP to the double-hybrid B2-PLYP, the correlation energy part computed at PT2 level enhances the binding character. On the other hand, PBE-based approaches generally overestimate of the binding energy. More generally in this example, no net improvement is noted going from semilocal to double-hybrid schemes.

As a second example, we selected a system of biological interest: the uracil dimer possessing two hydrogen-bonds (Figure 7). If all the considered density-functionals reproduce the binding behavior, here again non-empirical DHs provide the smallest errors (ranging between 1 and 3\%) while B3LYP error is around 13\%.

To illustrate the performance of functionals on systems dominated by dispersion interactions, the results obtained on the benzene-ethene and the stacked pyrazine dimers, reported in Figure 8, will be discussed. Analyzing the benzene-ethene potential energy curve, one can notice that the binding region is not accurately modeled by all functionals. Only double-hybrids, and particularly PBEQIDH, are able to reproduce the binding character while all others provide a fully repulsive potential energy curve. The same repulsive character in the binding region is clearly evident for the pyrazine dimer. Here again, only DHs succeed in quantitatively reproduce the attractive dispersion interactions. Finally, when considering inorganic heavy-metal complexes, for a dimer of hydrogen telluride only two DHs display the correct binding behavior while all of them qualitatively describe the plumbane hydrogen chloride interaction (Figure 9).

\subsection{Conformer Stabilities}

The quantitative evaluation of conformational stability is of particular interest when aiming at modeling large biological systems as in the case of drug discovery applications. An illustration of DFA performance for these types of interactions is reported in Figure 10. If all the considered DFAs generally provide a good accuracy, DHs bring a systematic improvement. For the worked out example (i.e. the phenylalanyl-glycyl-glycine tripeptide) reported in Figure 10, we can see that semilocal and global-hybrids lead to an even qualitatively wrong estimation of relative stability of the conformers while DHs are able to recover the correct energy ordering. 


\subsection{Vertical electronic transitions}

The GMTKN30 database is representative of a large number of ground-state properties and reactions. For the sake of completeness, we extended the assessment of DHs on excited states using a recently developed dataset composed by 80 organic molecules. ${ }^{53}$ This set probes both absorption and emission excitations. It contains some problematic cases for TD-DFT, such as excitations of cyanines dyes or transition with charge transfer character. This variety justifies the relative high error obtained for the reference method, PBE0 (about $0.2 \mathrm{eV}$ ). The results obtained with DHs are reported in Figure 11. It clearly appears that PBE-QIDH performs as well as PBE0, while PBE0DH is slightly better. The two DHs provide, however, significantly lower errors than PBE or even LC- $\omega$ PBE. The physics underpinning this behavior is difficult to rationalize, since the calculation of excited states for DHs is based on the replacement of the nonlocal correlation contribution by a CIS(D) correction, ${ }^{58}$ keeping the same weights as in the original functional. ${ }^{59}$

Nevertheless, these results clearly indicate that DHs perform at least as well as one of the most representative functional routinely used for the excited states.

\section{Fine tuning}

The absence of a fitting procedure confers an "all purposes" character to the non-empirical DHs, which we could define as a kind of aura mediocritas in DFA. Following the logic of "the best of two worlds”, further improvements of numerical accuracy could come from recent developments of WF methods. For instance, the orbital optimization scheme, i.e. the self-consistent minimization of the total energy with respect to changes in orbitals, has been recently applied to the PBE0-DH and QI-DH schemes ${ }^{18,19}$. In both cases, the modest impact found on the accuracy is counterbalanced by a significant increase of the computation times, making these methods unaffordable for larger systems, though interesting for difficult open-shell cases. Another possibility of improving the performance of the MP2 approach recently explored is the so-called spin-component scaling, where Same-Spin (SS) and Opposite-Spin (OS) contribution to the MP2 energy are scaled by an empirical factor. We have recently introduced the OS variant of the QIDH functional (SOS1-QIDH) ${ }^{60}$, where the SS contribution is fixed a priori and the OS is embedded in the GGA part. This approach is a computationally convenient alternative to reach the accuracy of the parent QIDH functional, without losing theoretical ground, as for the mentioned OO scheme.

Further works showed that improvement can be reached by relaxing some theoretical constraints. In particular, xDH-PBE0 approach, where all the parameters are optimized and PBE0 orbitals are used for the evaluation of the OS-MP2 contribution, is one of the best performing DHs. ${ }^{22,61}$ 


\section{Conclusion and Remarks}

The quest for better DFAs has undoubtedly leaded to the development of robust and reliable approaches contributing to the success of DFT. Among them, non-empirical functionals are particularly attracting for their well-defined physical background. Following the lines traced by the PBE approach, a GH (PBE0) and DH (QI-DH) have been proposed so that a whole family of nonempirical functionals, spanning on the highest rungs of the Perdew's quality scale, is now available. These functionals are not only competitive with parametrized approaches, but they also show how the increasing of the number of theoretical constrains leads to an improvement of their numerical performance. This fact further strengths the relevance of non-empirical functionals in the broader context of DFAs and, hopefully, will further encourage their development and routine application.

Looking into the physics underpinning their performance, it could be argued that all the ingredients necessary to cope with short and long-range electronic effects are present in DHs. Indeed, the (relatively) high HF exchange contribution plays a major role in giving correct reaction barriers, in reducing SIE and in providing accurate vertical transitions energies. Thermodynamics is mainly dominated by the chosen GGA approach, while the MP2 contribution finely tunes it and gives a substantial contribution to successfully deal with weak interactions. Of course, all these ingredients are also present in parametrized DHs, but the theoretical foundation of non-empirical functionals provides a solid physical and chemical ground releasing them from all problems related to any parametrized approach (domain of application, over-parametrization, single vs. multi set of parameters etc.).

Still one question may be asked: are we adding up DFT and WF problems? From the results obtained the answer is no, since there is a beneficial synergic effect of DF and WF contributions, as exemplified by the results obtained on the thermochemistry dataset (Figure 1). Here, the error on atomization energies of the PBE0-DH model are reported as function of the coefficients $a_{x}$ and $a_{c}$ in eqn(1). It is thus clear that DHs represent a substantial enhancement upon both the two limiting approaches (PBE and MP2).

Finally, if DHs are still computationally expensive, it is worthwhile to stress that they can benefit of all technical enhancements developed for speeding-up post-HF approaches, providing substantial hope for their routine application in a competitive way with respect to traditional GGA and GH approaches.

\section{Acknowledgements}

This project has received funding from the European Research Council (ERC) under the European Union's Horizon 2020 research and innovation programme (grant agreement No 648558). 


\section{Biographical Information}

Éric Brémond graduated from École Nationale Supérieure de Chimie de Montpellier (France) in 2009, and completed his Ph.D in Theoretical Chemistry from ChimieParisTech (France) in 2012. After two years as postdoctoral fellow at École Polytechnique Fédérale de Lausanne (Switzerland), he became junior researcher at Istituto Italiano di Tecnologia (Genoa, Italy). His main research interests concern the development and application of DFT and TDDFT

Ilaria Ciofini after a master degree in Chemistry at the University of Florence (Italy) obtained her doctoral degree in 2001 from the University of Fribourg (Switzerland). After two postdoctoral fellowships (Uni. Wurzburg, Germany and ChimieParisTech, France) she got a permanent CNRS scientific engineer position in 2004. Since 2010, she is CNRS Research Director at ChimieParisTech. Her research focus on the development and application of DFT approaches to the design and description of complex molecular systems.

Juan-Carlos Sancho-García received (2001) a Ph.D. degree in Quantum Chemistry at University of Alicante. After stays in Prague (Czech Republic) and Mons (Belgium), he returned (2005) to Alicante with a tenure-track 'Ramón y Cajal' senior research position, and later on (2010) become Professor of Physical Chemistry, developing teaching and research activities. His interest is mostly focused on DFT studies, including developments and applications to organic molecular semiconductors and weak interactions.

Carlo Adamo did all his studies at University Federico II, receiving a $\mathrm{PhD}$ in Theoretical Chemistry in 1995. Between 1993 and 2000 he was Assistant Professor at the University of Basilicata (Italy). In 2000, he moved to Chimie-ParisTech (France) where he is Full Professor in Theoretical Chemistry since 2004. In 2011 he was nominated senior member of the Institute Universitaire de France. His main research interests concern development of DFT approaches and applications to chemistry. 


\section{References}

[1] Perdew, J. P.; Ruzsinszky, A.; Constantin, L. A.; Sun, J.; Csonka, G. I. Some fundamental issues in ground-state density functional theory: A guide for the perplexed. J. Chem. Theory Comput. 2009, 5, 902-908.

[2] Adamo, C.; Barone, V. Toward reliable density functional methods without adjustable parameters: The PBE0 model. J. Chem. Phys. 1999, 110, 6158-6170.

[3] Ernzerhof, E.; Scuseria, G. E. Assessment of the Perdew-Burke-Ernzerhof exchange-correlation functional. J. Chem. Phys. 1999, 110, 5029-5036.

[4] Becke, A. D. Density-functional thermochemistry. III. The role of exact exchange. J. Chem. Phys. 1993, 98, 5648-5652.

[5] Barone, V.; Adamo, C. Theoretical study of direct and water assisted isomerization of formaldehyde radical cations. A comparison between density functional and post Hartree-Fock approaches, Chem. Phys. Lett. 1994, 224, 432-438

[6] Stephens, P. J.; Devlin, F. J.; Chabalowski, C. F.; Frisch, M. J. Ab Initio Calculation of Vibrational Absorption and Circular Dichroism Spectra Using Density Functional Force Fields. J. Chem. Phys. 1994, 98, 11623-11627.

[7] Yanai, T.; Tew, D.; Handy, N. A new hybrid exchange-correlation functional using the Coulomb-attenuating method (CAM-B3LYP). Chem. Phys. Lett. 2004, 393, 51-57.

[8] Vydrov, O. A.; Scuseria, G. E. Assessment of a long-range corrected hybrid functional. J. Chem. Phys. 2006, 125, 234109.

[9] Andersson, Y.; Langreth, D. C.; Lundqvist, B. I. van der Waals interactions in density functional theory. Phys. Rev. Lett. 1996, 76, 102-105.

[10] Cornaton, Y.; Stoyanova, A.; Jørgen H.; Jensen, A.; Fromager, E. Alternative separation of exchange and correlation energies in range-separated density-functional perturbation theory. Phys. Rev. A 2013, 88, 022516.

[11] Ángyán, J. G.; Gerber, I. C.; Savin, A; Toulouse, J.; van der Waals forces in density functional theory: Perturbational long-range electron-interaction corrections. Phys. Rev. A 2005, 72, 012510.

[12] Gorling, A; Levy, M. Correlation-energy functional and its high-density limit obtained from a coupling-constant perturbation expansion. Phys. Rev. B 1993, 47, 13105-13113.

[13] Mori-Sanchez, P.; Wu, Q.; Yang, W. Orbital-dependent correlation energy in densityfunctional theory based on second-order perturbation approach: Success and failure. J. Chem. Phys. 2005, 123, 062204.

[14] Ernzerhof, M. Construction of the adiabatic connection. Chem. Phys. Lett. 1996, 263, 499-506. 
[15] Zhao, Y.; Lynch, B. J.; Truhlar, D. G. Doubly Hybrid Meta DFT: New Multi-Coefficient Correlation and Density Functional Methods for Thermochemistry and Thermochemical Kinetics. $J$. Phys. Chem. A 2004, 108, 4786-4791.

[16] Grimme, S. Semiempirical hybrid density functional with perturbative second-order correlation. J. Chem. Phys. 2006, 124, 034108.

[17] Bartlett, R. J.; Lotrich, V. F.; Schweigert, I. V. Ab initio density functional theory: The best of both worlds? J. Chem. Phys. 2005, 123, 062205.

[18] Peverati, R.; Head-Gordon, M. Orbital optimized double-hybrid density functionals. J. Chem. Phys. 2013, 139, 024110.

[19] Sancho-García, J.C.; Pérez-Jiménez, A.J.; Savarese, M.; Brémond, E.; Adamo, C. Importance of Orbital Optimization for Double-Hybrid Density Functionals: Application of the OO-PBE-QIDH Model for Closed- and Open-Shell Systems J. Phys. Chem. A 2016, 120, 1756-1762.

[20] Kozuch, S.; Gruzman, D.; Martin, J. M. L. DSD-BLYP: A General Purpose Double Hybrid Density Functional Including Spin Component Scaling and Dispersion Correction. J. Phys. Chem. C 2010, 114, 20801.

[21] Zhang, I. Y.; Xu, X.; Jung, Y.; Goddard, W. A. A fast doubly hybrid density functional method close to chemical accuracy using a local opposite spin ansatz. Proc. Natl. Acad. Sci. USA 2011, 108, 19896-19900.

[22] Zhang, I. Y.; Su, N. Q.; Brémond, E.; Adamo, C.; Xu, X. Doubly hybrid density functional xDH-PBE0 from a parameter-free global hybrid model PBE0. J. Chem. Phys. 2012, 136, 174103.

[23] Zhang, I. Y.; Su, N. Q.; Brémond, E.; Adamo, C.; Xu, X. "Response to 'Comment on Doubly hybrid density functional xDH-PBE0 from a parameter-free global hybrid model PBE0’” [J. Chem. Phys. 143, 187101 (2015). J. Chem. Phys. 2015, 143, 187102.

[24] Zhang, Y.; Xu, X.; Goddard, W. A. Doubly hybrid density functional for accurate descriptions of non-bonded interactions, thermochemistry, and thermochemical kinetics. Proc. Natl. Acad. Sci. USA 2009, 106, 4963-4968.

[25] Harris, J. Adiabatic-connection approach to Kohn-Sham theory. Phys. Rev. A 1984, 29, 1648.

[26] Sharkas, K.; Toulouse, J.; Savin, A. Double-hybrid density-functional theory made rigorous. J. Chem. Phys. 2011, 134, 064113.

[27] Toulouse, J.; Sharkas, K.; Brémond, E.; Adamo, C. Rationale for a new class of double-hybrid approximations in density-functional theory. J. Chem. Phys. 2011, 135, 101102.

[28] Fromager, E. Rigorous formulation of two-parameter double-hybrid density-functionals. $J$. Chem. Phys. 2011, 135, 244106.

[29] Brémond, E.; Adamo, C. Seeking for parameter-free double-hybrid functionals: the PBE0-DH model. J. Chem. Phys. 2011, 135, 024106. 
[30] Brémond, E.; Sancho-Garcia, J. C.; Pérez-Jiménez, A. J.; Adamo, C. Double-hybrid functionals from adiabatic-connection: The QIDH model. J. Chem. Phys. 2014, 141, 031101.

[31] Bousquet, D.; Brémond, E.; Sancho-Garcia, J. C.; Ciofini, I.; Adamo, C. Is There Still Room for Parameter Free Double Hybrids? Performances of PBE0-DH and B2PLYP over Extended Benchmark Sets. J. Chem. Theory. Comput. 2013, 9, 3444.

[32] Perdew, J. P.; Burke, K.; Ernzerhof, M. Generalized Gradient Approximation Made Simple. Phys. Rev. Lett. 1996, 77, 3865.

[33] Tao, J. M.; Perdew, J. P.; Staroverov, V. N.; Scuseria, G. E.Climbing the density functional ladder: Nonempirical meta-generalized gradient approximation designed for molecules and solids. Phys. Rev. Lett. 2008, 91, 146401.

[34] Becke, A. D. A new mixing of Hartree-Fock and local density-functional theory. J. Chem. Phys. 1993, 98, 1372-1377.

[35] Perdew, J. P.; Ernzerhof, M.; Burke, K. Rationale for mixing exact exchange with density functional approximations. J. Chem. Phys. 1996, 105, 9982-9985.

[36] Adamo, C.; Barone, V. Toward reliable adiabatic connection models free from adjustable parameters. Chem. Phys. Lett. 1997, 274, 242.

[37] Jacquemin, D; Wathelet, V.; Perpète, E. A.; Adamo, C. Extensive TD-DFT benchmark: singlet-excited states of organic molecules. J. Chem. Theory Comput. 2009, 5, 2420-2435.

[38] Ciofini, I.; Adamo, C.; Barone, V. Complete structural and magnetic characterization of biological radicals in solution by an integrated quantum mechanical approach: glycyl radical as a case study. J. Chem. Phys. 2004, 121, 6710.

[39] Wu, X.; Selloni, A.; Car, R. Order-N implementation of exact exchange in extended insulating systems. Phys. Rev. B 2009, 79, 085102.

[40] Zhang, C.; Donadio, D.; Gygi, F.; Galli, G. First principles simulations of the infrared spectrum of liquid water using hybrid density functionals. J. Chem. Theory Comp. 2011, 7, 1443.

[41] Curtiss, L. A.; Raghavachari, K.; Trucks, G. W.; Pople, J. A. Gaussian-2 theory for molecular energies of first and second-row compounds. J. Chem. Phys. 1991, 94, 7221-7230.

[42] Guido, C. A.; Brémond, E.; Adamo, C.; Cortona, P. One third: a new recipe for the PBE0 paradigm. J. Chem. Phys. 2013, 138, 021104.

[43] Ren, X. ; Tkatchenko, A. ; Rinke, P. ; Scheffler, M. Beyond the Random-Phase Approximation for the Electron Correlation Energy: The Importance of Single Excitations Phys. Rev. Lett. 2011, 106, 153003

[44] Brémond, E.; Savarese, M.; Pérez-Jiménez, A. J.; Sancho-García, J. C.; Adamo, C. Systematic Improvement of Density Functionals through Parameter-Free Hybridization Schemes. J. Phys. Chem. Lett. 2015, 6, 3540-3545. 
[45] Cohen, A. J.; Mori-Sanchez, P.; Yang, W. Assessment and formal properties of exchangecorrelation functionals constructed from the adiabatic connection. J. Chem. Phys. 2007, 127, 034101

[46] Su, N. Q.; Xu, X. Construction of a parameter-free doubly hybrid density functional from adiabatic connection. J. Chem. Phys. 2014, 140, 18A512.

[47] Kim, J.; Jung Y. Analytical double-hybrid density-functional based on the polynomial series expansion of adiabatic connection: a quadratic approximation. J. Chem. Theory Comput. 2015, 11, 45-54.

[48] Curtiss, L. A.; Raghavachari, K.; Redfern, P. C.; Pople, J. A. Assessment of Gaussian-3 and density-functional theories for a larger experimental test set. J. Chem. Phys. 2000, 112, 7374-7383.

[49] Peverati, R.; Truhlar, D. G. Quest for a universal density functional: the accuracy of density functionals across a broad spectrum of databases in chemistry and physics. Phil. Trans. R. Soc. A. 2014, 372, 20120476.

[50] Gráfová, L.; Pitoňák, M.; Řezáč, J.; Hobza, P. Comparative Study of Selected Wave Function and Density Functional Methods for Noncovalent Interaction Energy Calculations Using the Extended S22 Data Set. J. Chem. Theory Comput. 2010, 6, 2365-2376.

[51] Rezac, J.; Riley, K.; Hobza, P. S66: A Well-balanced Database of Benchmark Interaction Energies Relevant to Biomolecular Structures. J. Chem. Theory Comp., 2011, 7, 2427-2438.

[52] Goerigk, L.; Grimme, S. Efficient and Accurate Double-Hybrid-Meta-GGA Density Functionals-Evaluation with the Extended GMTKN30 Database for General Main Group Thermochemistry, Kinetics, and Noncovalent Interactions. J. Chem. Theory Comput. 2010, 6, 107126.

[53] Jacquemin, D.; Duchemin, I.; Blase, X. 0-0 Energies Using Hybrid Schemes: Benchmarks of TD-DFT, CIS(D), ADC(2), CC2, and BSE/GW formalisms for 80 Real-Life Compounds. J. Chem. Theory. Comput. 2015, 11, 5340-5359.

[54] Becke, A. D. Density-functional exchange-energy approximation with correct asymptoticbehavior. Phys. Rev. A 1988, 38, 3098-100.

[55] Lee, C.; Yang, W.; Parr, R. G. Development of the Colle-Salvetti correlation-energy formula into a functional of the electron density. Phys. Rev. B 1988, 37, 785-89.

[56] Cohen, A. J.; Mori-Sanchez, P.; Yang, W. Insights into current limitations of density functional theory. Science 2008, 321, 792.

[57] Brémond, E.; Ciofini, I.; Adamo, C. Gradient-regulated connection-based correction for the PBE exchange: the PBEtrans model, Mol. Phys. 2016, 114, 1059-1065.

[58] Head-Gordon, M.; Rico, R. J.; Oumi, M.; Lee, T. J. A doubles correction to electronic excited states from configuration interaction in the space of single substitutions. Chem. Phys. Lett. 1994, 219, 21. 
[59] Grimme, S.; Neese, F. Double-hybrid density functional theory for excited electronic states of molecules. J. Chem. Phys. 2007, 127, 154116.

[60] Brémond, E.; Savarese, M.; Sancho-García, J.C.; Pérez-Jiménez, A.J.; Adamo, C., Quadratic integrand double-hybrid made spin-component-scaled, J. Chem. Phys. 2016, 144, 124104.

[61] Brémond, E.; Savarese, M.; Su, N.Q.; Pérez-Jiménez, A.J.; Xu, X.; Sancho-García, J.C.; Adamo, C. Benchmarking Density Functionals on Structural Parameters of Small-/Medium-Sized Organic Molecules J. Chem. Theory Comp., 2016, 12, 459-465. 


\section{Figure captions}

Figure 1. Mean absolute deviations for the G2-148 dataset as function of the fraction of HFexchange $\left(a_{x}\right.$, left), and of the fractions of HF-exchange and PT2 correlation $\left(a_{x}, a_{c}\right.$, right). PBE0 corresponds to $a_{x}=0.25, a_{c}=0.0$; PBE0-DH to $a_{x}=0.50, a_{c}=0.15$, PBE-QIDH to $a_{x}=0.70, a_{c}=0.0$ and MP2 to $a_{x}=1.0, a_{c}=1.0$. The $6-311+\mathrm{G}(3 d f, 2 p d)$ basis set was used.

Figure 2. Mean absolute deviations for the atomization and decomposition (left) and the adiabatic processes (right) databases (see reference 45 for details). The def2-QZVP basis set was used.

Figure 3. Mean absolute deviations for the self-interaction error database (SIE11, right), evaluated with the def2-QZVP basis set (see reference 45 for details). The $\mathrm{H}_{2}{ }^{+}$dissociation (left) was computed with the aug-cc-pVQZ basis set.

Figure 4. Mean absolute deviations for the barrier height database (right) from the GMTKN30 dataset (see reference 45 for details). Energy differences between transition state and reactants computed on two $\mathrm{S}_{\mathrm{N}} 2$ reactions (left), fluoride attack on 1-chloromethane and chloride attack on 1fluoromethane. The def2-QZVP basis set was used .

Figure 5. Mean absolute deviations for the reaction database (left) built from the GMTKN30 dataset (see reference 45 for details). Example of reaction energy (right) computed for the isomerization of 2,2-dimethylpropane into pentane. The def2-QZVP basis set was used.

Figure 6. Mean absolute deviations computed for the weak interaction database from the GMTKN30 dataset (see reference 45 for details). The def2-QZVP basis set was used.

Figure 7. Water dimer dissociation (left) computed with theaug-cc-pVTZ basis set. Reference $\mathrm{CCSD}(\mathrm{T}) / \mathrm{CBS}$ energies are taken from the $\mathrm{S} 66 \times 8$ repository ${ }^{54}$. Example of hydrogen binding energies (right) computed at the def2-QZVP level on an uracil dimer.

Figure 8. Benzene-ethene dissociation (left) computed with the aug-cc-pVTZ basis set. Reference $\mathrm{CCSD}(\mathrm{T}) / \mathrm{CBS}$ energies are taken from S66 $\times 8$ repository ${ }^{54}$. Example of $\pi$-stacking energies (right) computed at def2- QZVP level on a pyrazine dimer.

Figure 9. Examples of weak interaction energy computed on two inorganic transition metal complexes: dimer of hydrogen telluride (left) and plumbane - hydrogen chloride interaction (right). The def2-QZVP basis set was used.

Figure 10. Mean absolute deviations for the conformer database (left) built from the GMTKN30 dataset (see reference 45 for details). Example of conformer stability (right) computed for the phenylalanyl-glycyl-glycine tripeptide case of study. The def2-QZVP basis set was used.

Figure 11. Mean absolute deviations for the excitation dataset (structures and reference values from reference 46). The 6-31+G(d) basis set was used for PBE, PBE0 and LC- $\omega$ PBE calculations, and the def2-TZVP basis set for PBE0-DH and PBE-QIDH. 
Figure 1

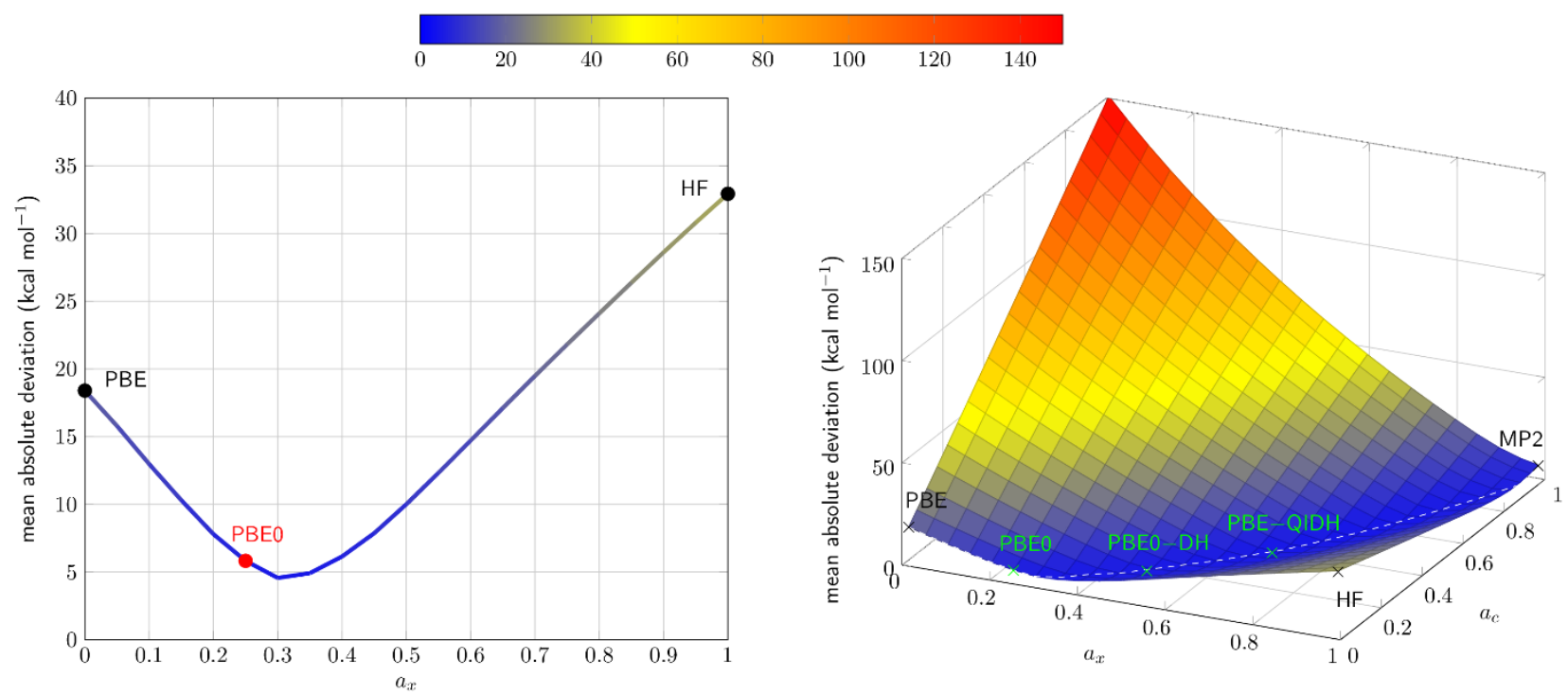


Figure 2
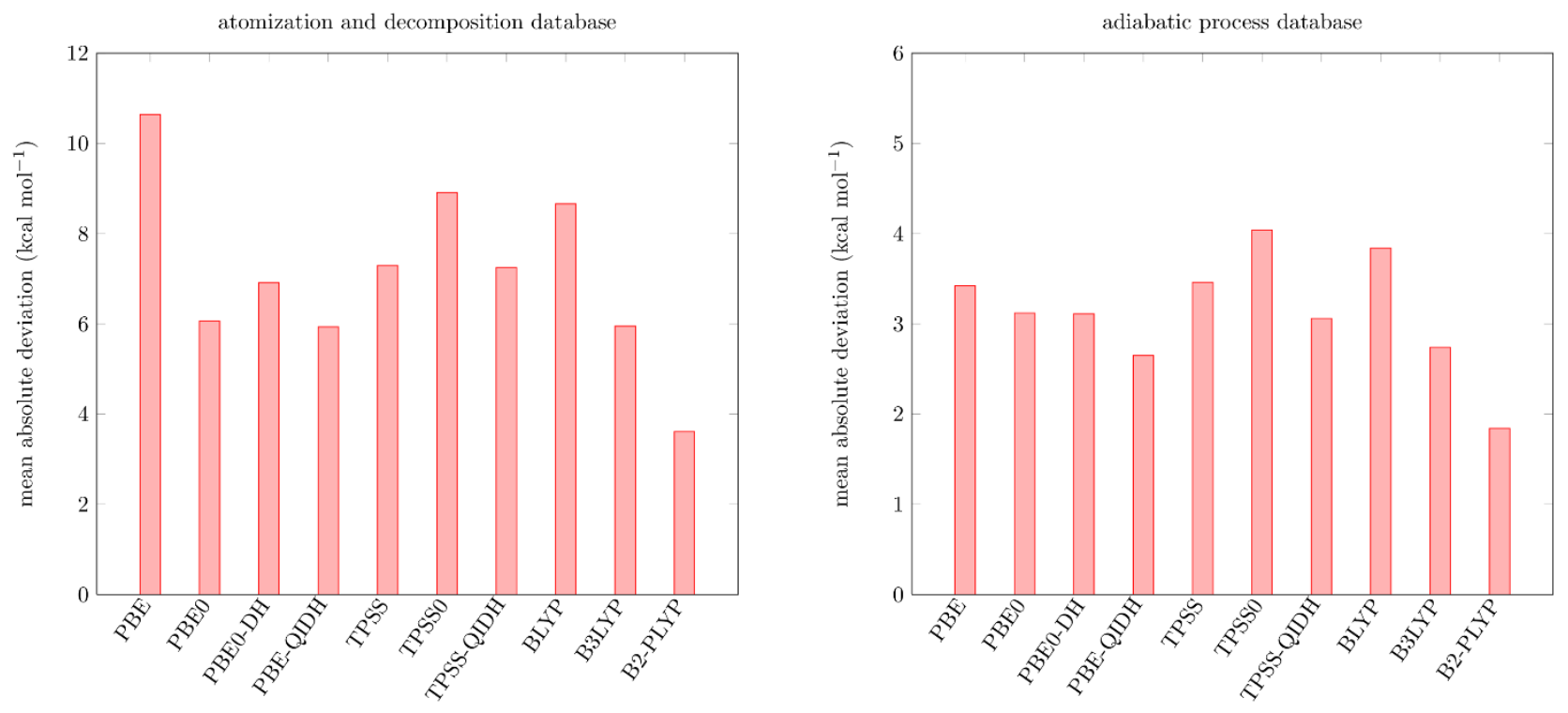
Figure 3
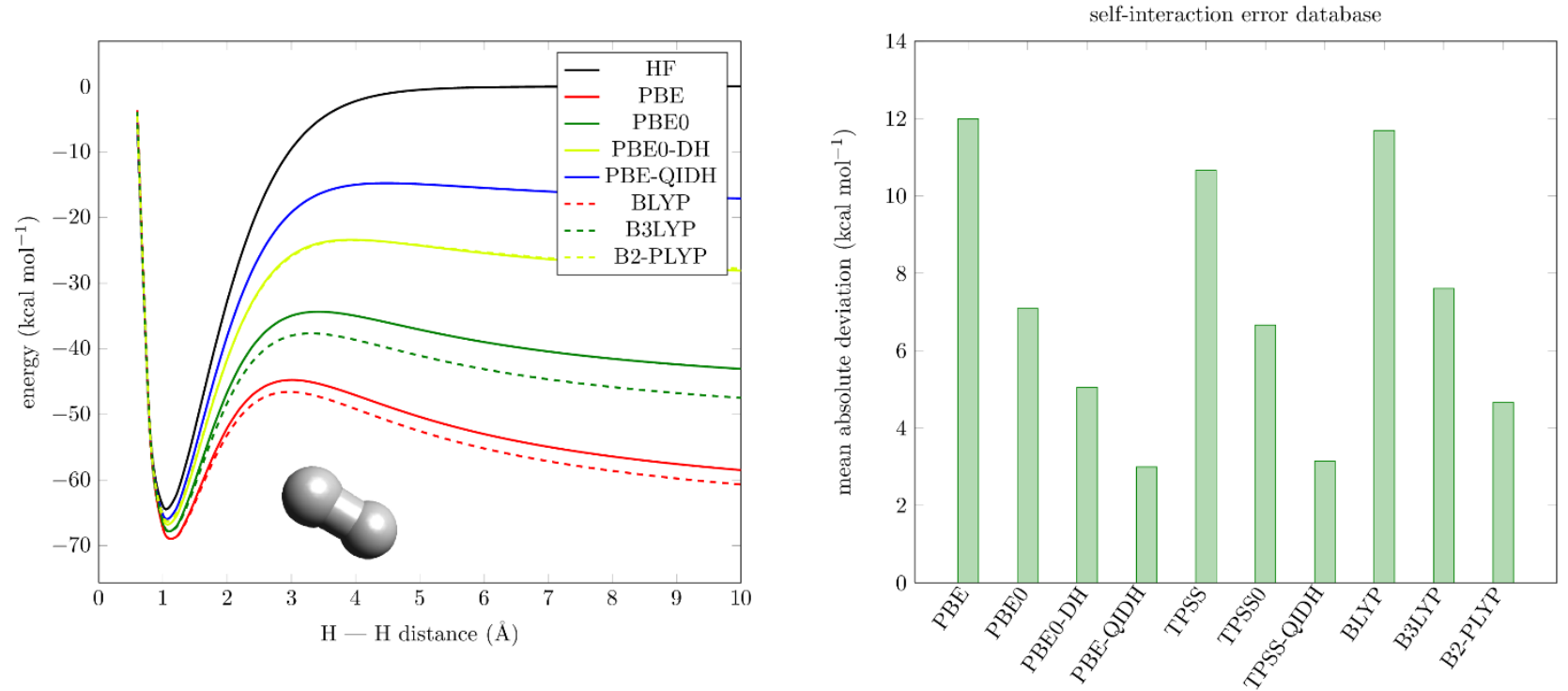
Figure 4
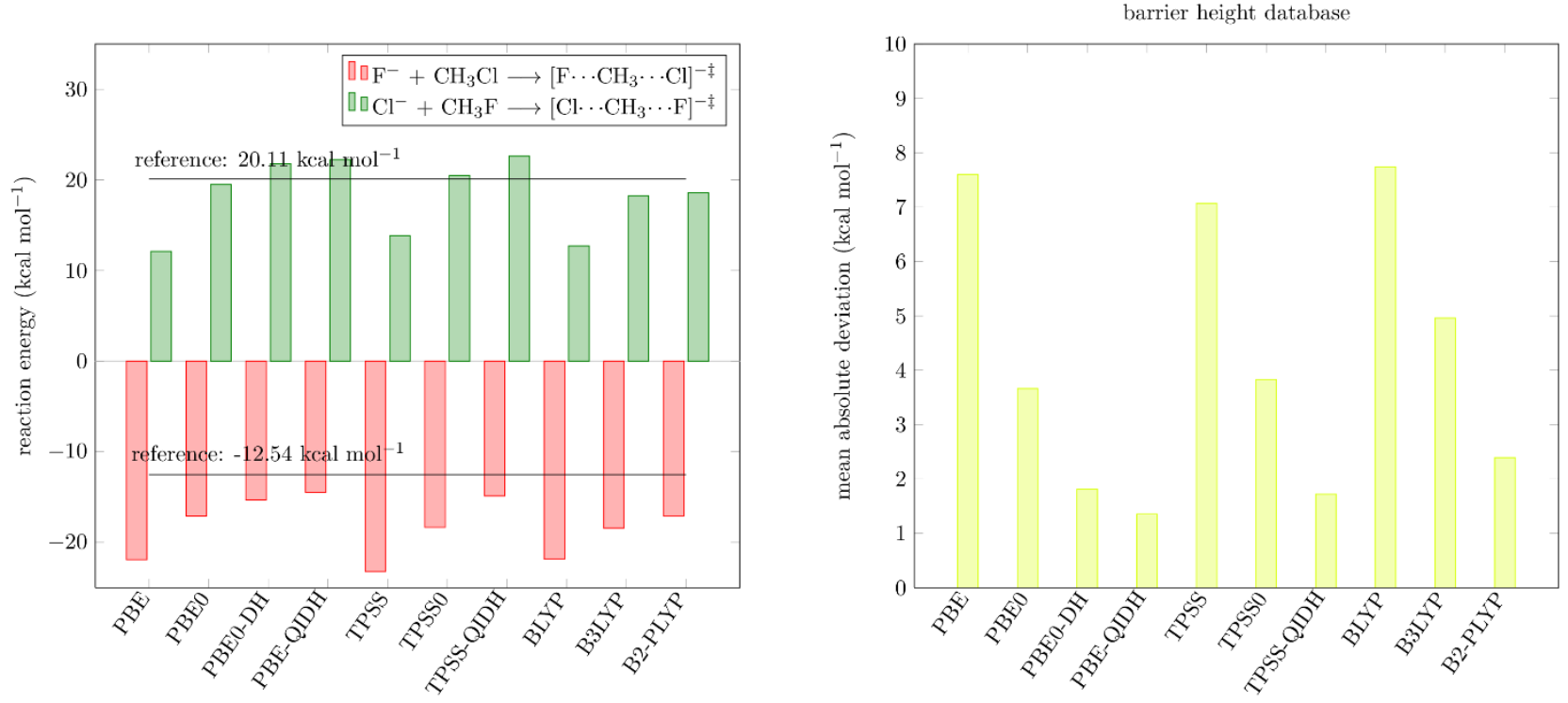
Figure 5
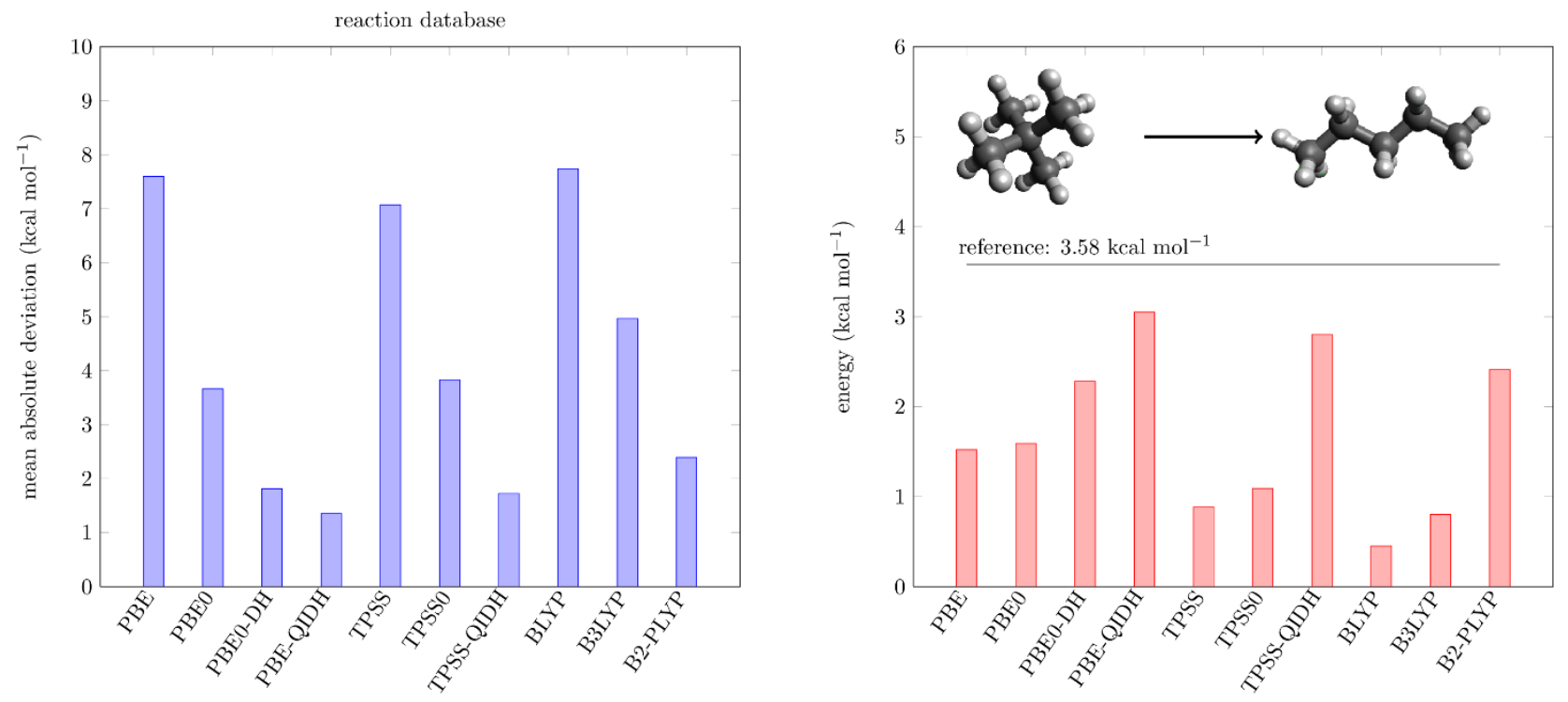
Figure 6

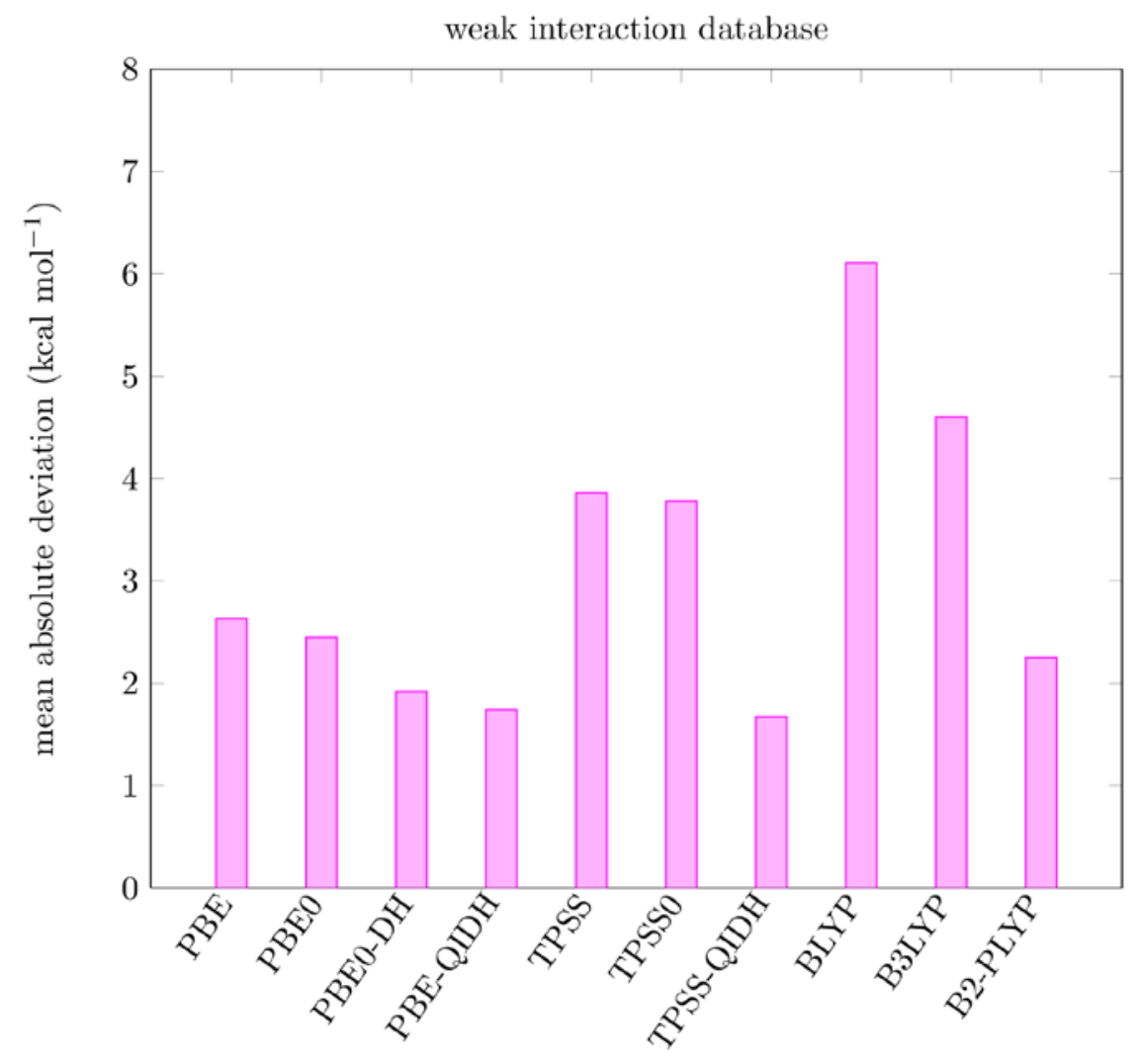


Figure 7
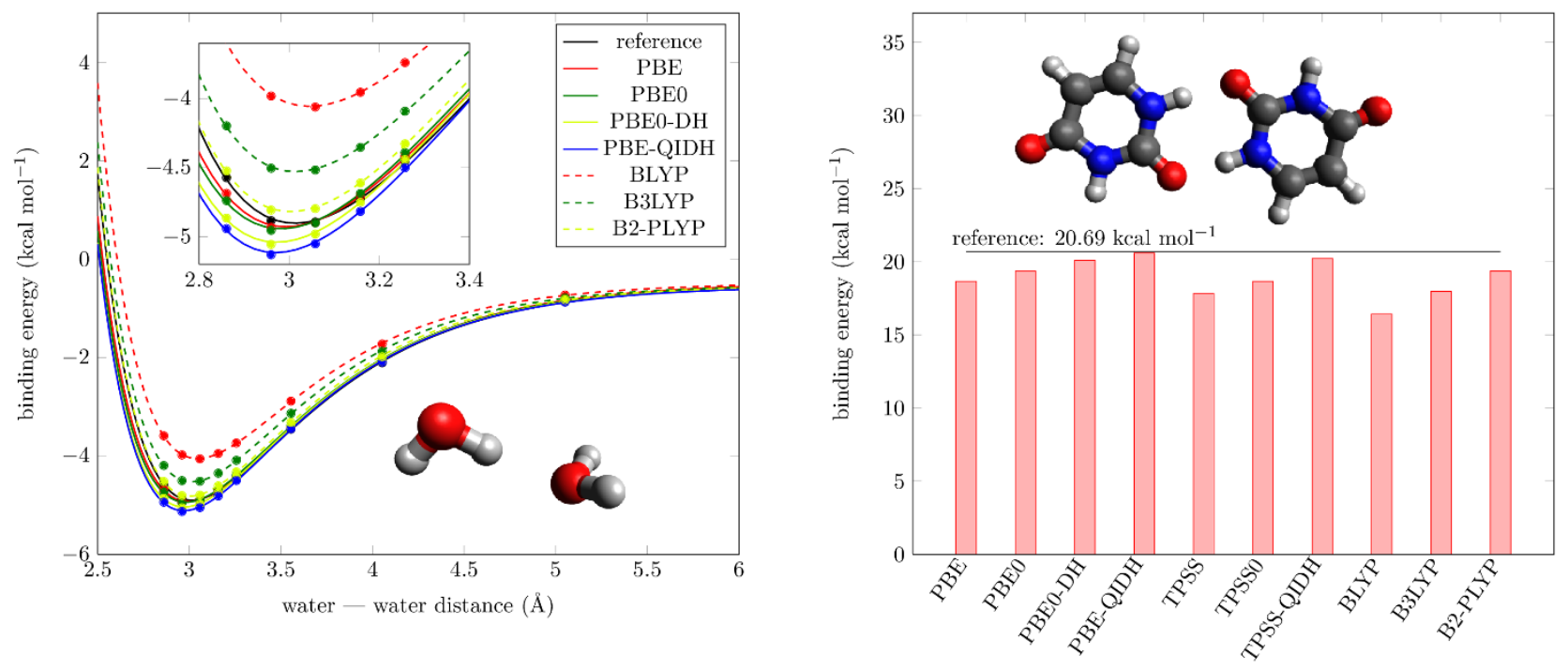
Figure 8
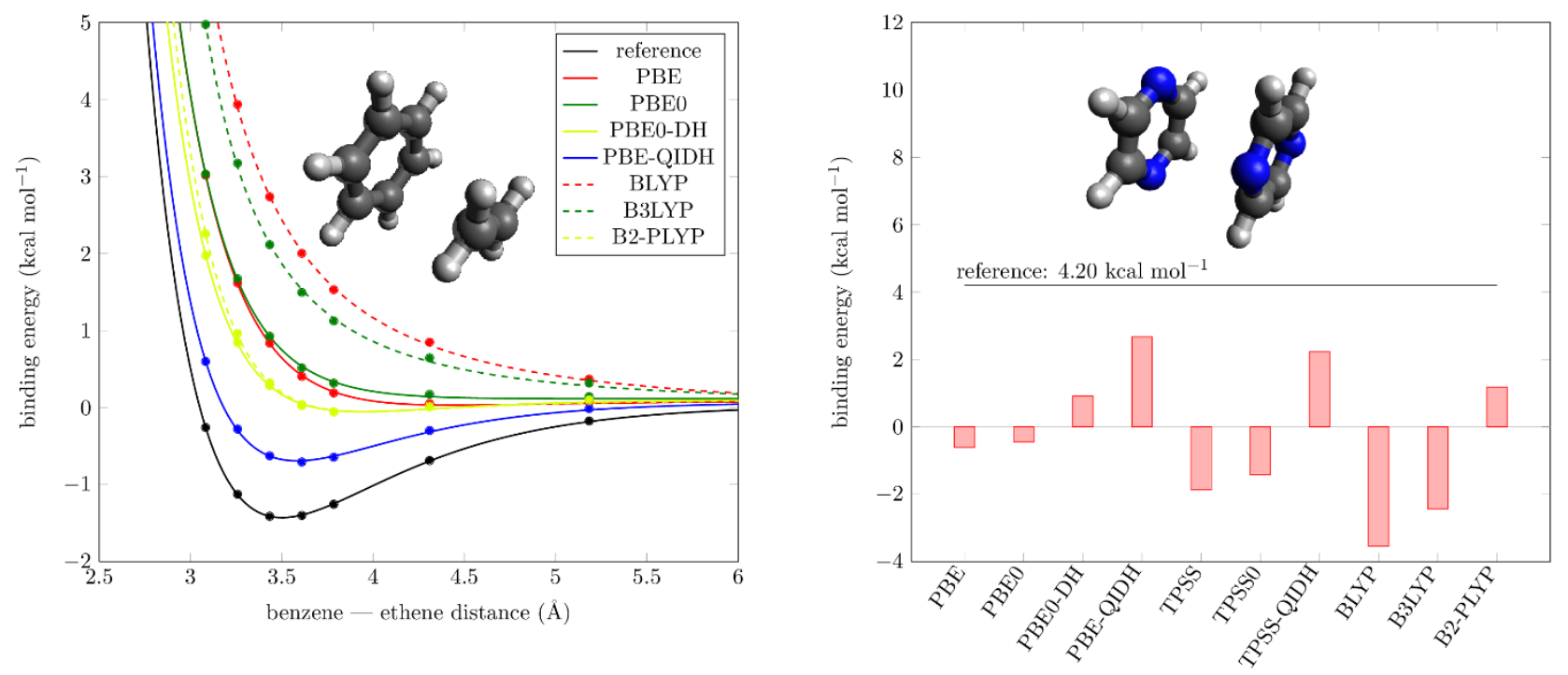
Figure 9
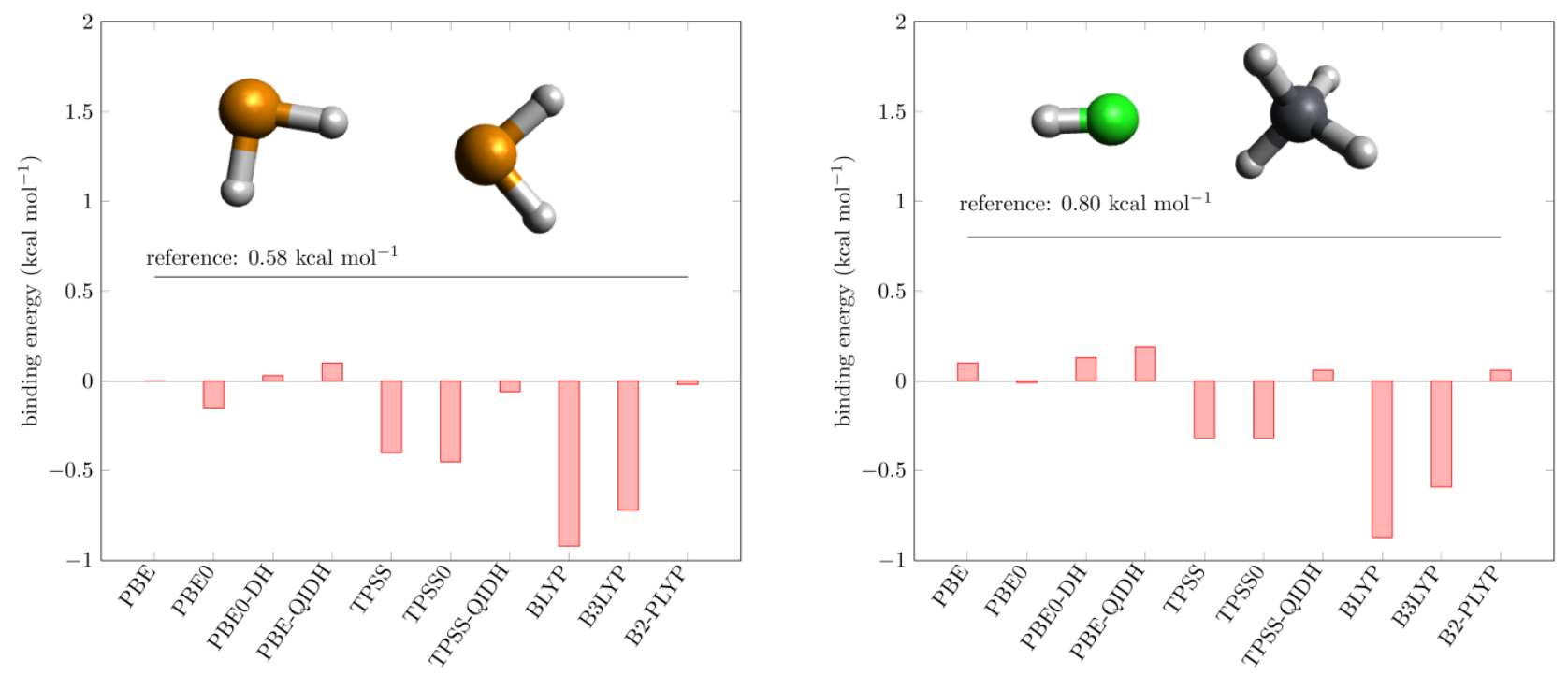
Figure 10
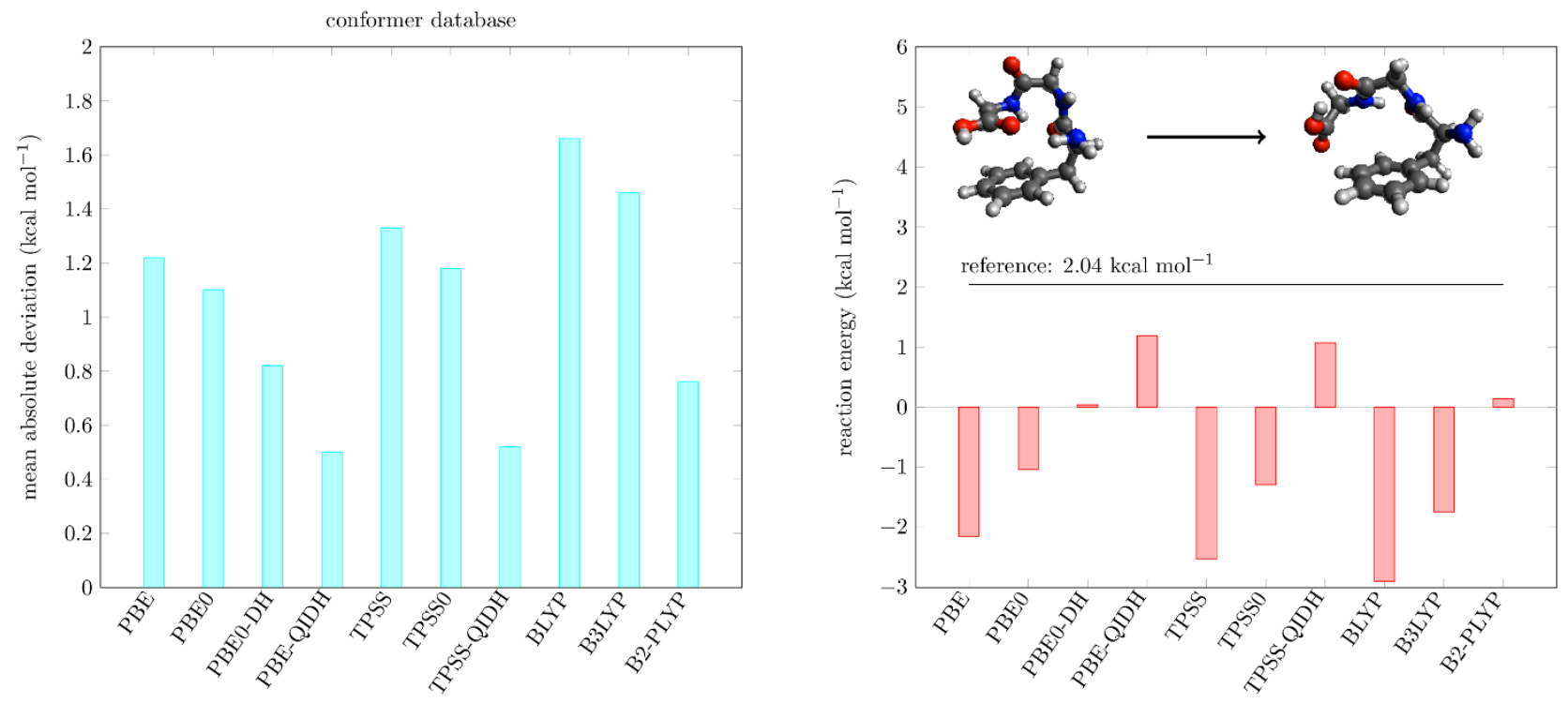
Figure 11

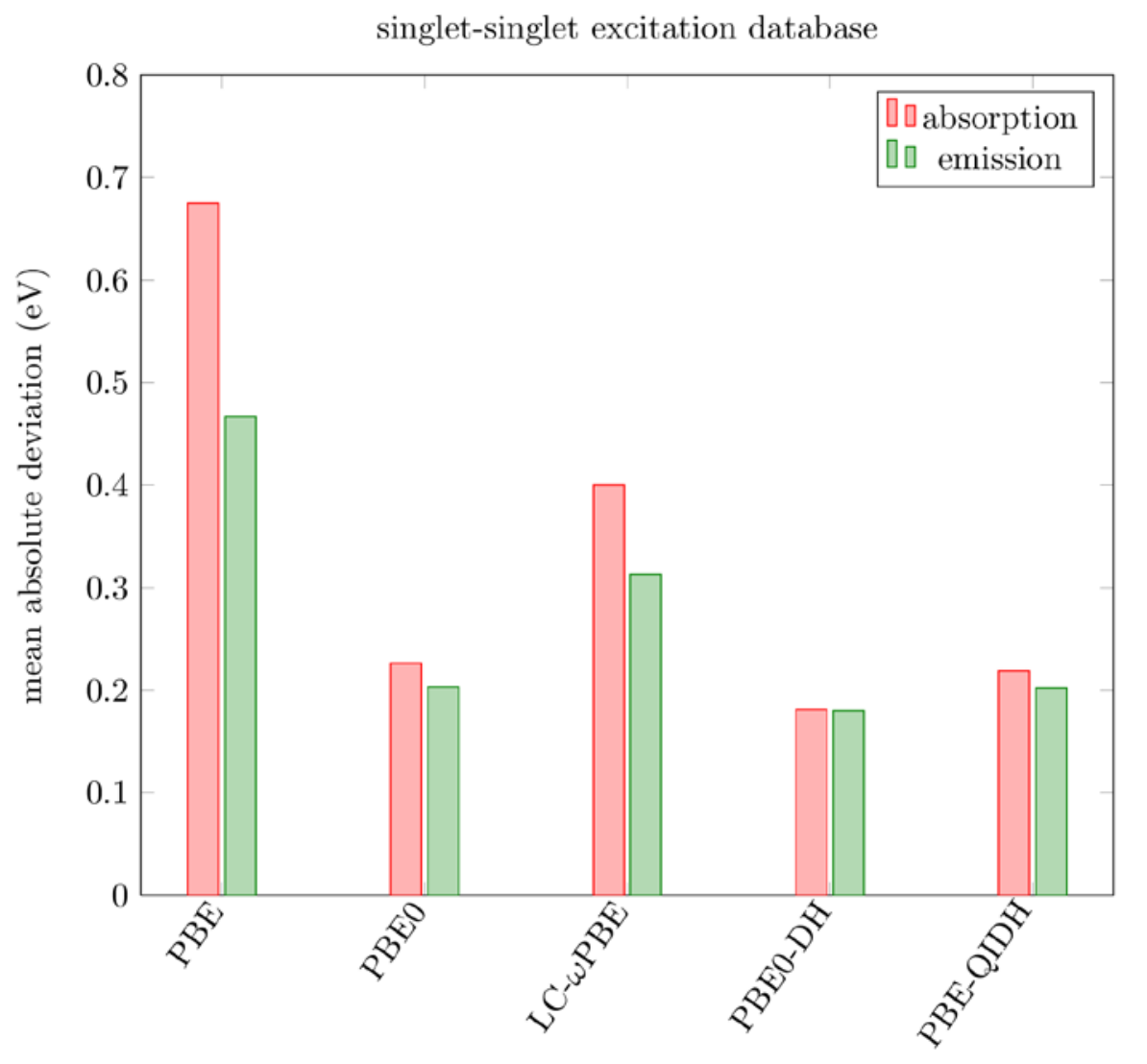

